\title{
Covid-19: Critically ill patients treated with arthritis drug tocilizumab show improved outcomes, researchers report
}

\section{Jacqui Wise}

The rheumatoid arthritis drug tocilizumab seems to improve outcomes in patients with covid-19 who are the most severely ill, show early results released from the Remap-Cap international platform trial.

The researchers reported that critically ill patients receiving tocilizumab were more likely to improve than patients who received no immune modulator (odds ratio 1.87). "These are very exciting and encouraging results," said the chief investigator, Anthony Gordon, consultant in intensive care medicine at Imperial College Healthcare NHS Trust, at a Science Media Centre briefing on 19 November.

He said that this was a bigger result than the 1.43 odds ratio seen with the steroid dexamethasone reported earlier by the trial.

However, the results are based on a combined outcome measure of survival and the length of time patients need organ support, such as a breathing machine, and the relative contribution of each has not yet been analysed. The results were made available by press release and have not yet been peer reviewed. ${ }^{1}$

Previous studies on the immune modulator tocilizumab have produced mixed results. A recent trial conducted among inpatients and reported in the New England Journal of Medicine did not find a survival benefit in those taking tocilizumab; however, they were less severely ill than those in the Remap-Cap trial. ${ }^{2}$

\section{Best combination}

The latest analysis was carried out by a statistical analysis committee separate from the trial investigators and reviewed by an independent data and safety monitoring board. The board advised the trial to stop recruiting patients into the no immune modulator arm on the basis of the findings.

The analysis included data from the first 303 patients randomly assigned to receive immune modulation treatments (tocilizumab, sarilumab, anakinra, or interferon) or no immune modulator. The relative benefits of tocilizumab as compared with the other immune modulator drugs have not yet been reported.

Patients were given the immune modulation treatment on the first day of becoming seriously unwell and moving into intensive care. Some of the patients will also have previously received the steroid dexamethasone, but those recruited early in the trial, before it became standard practice, will not.

Gordon said, "It is unlikely that any single drug will work on its own. This is why we need to analyse the best combination of treatments." He added that the team were working hard to analyse the results on 800 patients and hoped to publish the full results in the next few weeks.

Tocilizumab is given by intravenous infusion and works by blocking the cytokine interleukin 6 . "Seriously ill patients have a lot of lung inflammation, and the drug works through its anti-cytokine effect,” Gordon explained.

The drug costs $£_{500-£ 1000}\left(€_{558-€ 1117}\right.$; $\$ 661-\$ 1323$ ) and is much more expensive than dexamethasone but cheaper than a longer stay in intensive care, he added.

\section{Mixed results reported}

The latest analysis also showed that the antiviral drug combination lopinavir-ritonavir was ineffective and provided no additional benefit to patients critically ill with covid-19 when compared with those who did not receive the drug (odds ratio 0.67). The Recovery trial also found that lopinavir-ritonavir did not improve survival of patients with covid-19 in hospital, although this was among less seriously ill patients. ${ }^{3}$

The Remap-Cap trial has enrolled over 2000 patients in 15 countries who are randomly assigned to multiple treatment combinations. It is also evaluating therapeutic anticoagulation, convalescent plasma, vitamin $\mathrm{C}$, and macrolides. The study is supported in the UK by the National Institute for Health Research.

Peter Horby, professor of emerging infectious diseases and global health in the Nuffield Department of Medicine at the University of Oxford, commented, "The results so far on tocilizumab have been mixed, with four randomised controlled trials having reported results, of which two were negative and two were positive. None have reported a mortality benefit at day 28 or 30 . The results from Remap-Cap add to this evidence base, and I eagerly look forward to seeing the full results."

Stephen Evans, professor of pharmacoepidemiology at the London School of Hygiene and Tropical Medicine, said, "The size of the beneficial effect reported in this trial does not appear that high, and it combines survival with need for intense support. Therefore, I think these results need to be treated with caution at this early stage."

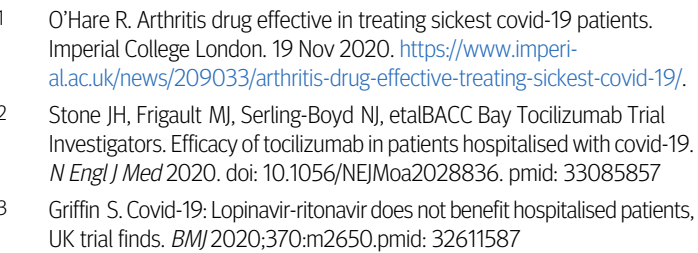


determined by BMJ. You may use, download and print the article for any lawful, non-commercial purpose (including text and data mining) provided that all copyright notices and trade marks are retained. 